\title{
Perfil físico-funcional e psicológico de idosos frequentadores do Rio Ar Livre
}

\author{
Physical function and psychological profiles of elderly \\ users of Rio Ar Livre
}

Helio Lemos Furtado ${ }^{1}$

Gabriela Venturini²

Elizabeth Rose Assumpção Harris ${ }^{3}$

Paulo de Tarso Veras Farinatti ${ }^{4}$

Nádia Souza Lima da Silva ${ }^{5}$

Endereço para Correspondência:

Nádia Souza Lima da Silva

Rua São Francisco Xavier, 524, sala 8121F

20.550-900 - Rio de Janeiro - RJ [Brasil]

nadiaslimas@gmail.com
1 Curso de Educação Física, Escola da Saúde e do Meio Ambiente - Universidade Castelo Branco - UCB. Rio de Janeiro, RJ - Brasil.

ORCID: https://orcid.org/0000-0002-2215-4102

2 Programa de Pós-Graduação em Ciências do Exercício e do Esporte, Instituto de Educação Física - Universidade do Estado do Rio de Janeiro - UERJ. Rio de Janeiro, RJ - Brasil. ORCID: https://orcid.org/0000-0001-5250-3222

3 Programa de Pós-Graduação em Ciências do Exercício e do Esporte, Instituto de Educação Física - Universidade do Estado do Rio de Janeiro - UERJ. Rio de Janeiro, RJ - Brasil. ORCID: https://orcid.org/0000-0002-0502-6846

4 Programa de Pós-Graduação em Ciências do Exercício e do Esporte, Instituto de Educação Física - Universidade do Estado do Rio de Janeiro - UERJ. Programa de PósGraduação em Ciências da Atividade Física - Universidade Salgado de Oliveira - UNIVERSO. Rio de Janeiro, RJ - Brasil. ORCID: https://orcid.org/0000-0003-2463-1280

5 Programa de Pós-Graduação em Ciências do Exercício e do Esporte, Instituto de Educação Física - Universidade do Estado do Rio de Janeiro - UERJ. Rio de Janeiro, RJ - Brasil. ORCID: https://orcid.org/0000-0003-0617-0521

\section{Resumo}

Introdução: A fim de desenvolver a saúde e a qualidade de vida, especialmente da população idosa, a Prefeitura da Cidade do Rio de Janeiro implementou o projeto Academia Rio Ar Livre (RAL) nos espaços públicos da cidade. Para acompanhamento longitudinal da repercussão desse projeto é necessário mapear a condição funcional e psicológica dos idosos inscritos nas diversas RALs. Objetivos: Assim, neste estudo, objetivou-se analisar a aptidão físico-funcional e psicológica de idosos frequentadores das RAL/RJ. Métodos: Selecionaram-se 294 voluntários de ambos os sexos $(68,6 \pm 6,6$ anos), que responderam o questionário de Escala de Depressão Geriátrica e o de Qualidade de Vida (WHOQOL-OLD) e realizaram a bateria de aptidão funcional de Rikli e Jones. Resultados: Verificou-se que os participantes possuíam níveis recomendados de aptidão funcional, alto nível de qualidade de vida e não apresentavam quadro de depressão. Conclusão: As RALs parecem contribuir para a manutenção da aptidão físico-funcional e psicológica de idosos.

Descritores: Qualidade de vida. Saúde. Idosos.

\begin{abstract}
Introduction: The City of Rio de Janeiro has implemented the "Academia Rio Ar Livre (RAL)" project in public spaces with the intent of promoting health and quality of life, especially for elderly people. In order to longitudinally monitor the repercussion of this project, it is necessary to map the functional and psychological conditions of elderly people enrolled in the RALs. Objectives: Thus, this study aimed to analyze the physical function and psychological profiles of elderly people who use the Rio de Janeiro RALs. Method: Two hundred ninety-four volunteers of both sexes, with an average age of $68.6 \pm 6.6$ years, were selected to respond to both the Geriatric Depression Scale and the (WHOQOL-OLD) quality of life questionnaires. They also took the Rikli and Jones Senior Fitness Test. Results: The participants possessed recommended levels of functional fitness and high levels of quality of life, showing no signs of depression. Conclusion: The RALs appear to contribute to the maintenance of physical, functional and psychological fitness of elderly people.
\end{abstract}

Keywords: Quality of life. Health. Elderly. 


\section{Introdução}

Projeções estimam que, em 2050, haverá na composição da população mundial 2 bilhões $(20 \%)$ de habitantes idosos. Destes, mais de $60 \%$ estarão vivendo em países em desenvolvimento como o Brasil ${ }^{1}$. Em razão disso, cresce a importância de iniciativas para preservar a saúde e qualidade de vida em idades avançadas. Nesse sentido, existe consenso de que a prática regular de atividade física é fator importante, dadas suas relações com a preservação da autonomia funcional e por auxiliar na prevenção e controle de muitas doenças crônico-degenerativas ${ }^{2}$.

Diante do exposto, políticas públicas que favoreçam o engajamento de idosos em programas de exercício físico pode ser uma boa fórmula para desenvolver a saúde e a qualidade de vida dessa parcela da população. Com esse objetivo, a Prefeitura da Cidade do Rio de Janeiro implementou o projeto Academia Rio Ar Livre (RAL), inicialmente chamado de Academia da Terceira Idade (ATI), cujo programa consiste em promover o exercício físico utilizando máquinas articuladas que não dispõem de regulagens para aumento progressivo da carga, com o intuito de desenvolver a força muscular e a prática de exercício aeróbio, preferencialmente de idosos, nos espaços públicos da cidade ${ }^{3}$. Por meio da facilitação do acesso à prática de exercícios neuromusculares e cardiorrespiratórios, a Prefeitura pretende aumentar as chances de preservação da autonomia e independência dos idosos, contribuindo para sua qualidade de vida.

A oferta de atividades físicas nas RALs dá-se com base em metodologia específica. Apesar de os equipamentos não possuírem regulagens de carga e se localizarem em praças públicas, com acesso gratuitamente franqueado à população, tenta-se controlar o volume e a intensidade dos exercícios, bem como os resultados alcançados. Nesse contexto, uma relevante característica do projeto é a oferta de apoio profissional para supervisionar as ativi- dades, a fim de garantir continuidade de progressão das cargas e quantificação dos efeitos do programa ${ }^{4}$.

É compreensível, portanto, a necessidade de se mapear a condição funcional e psicológica dos idosos inscritos nas diversas RALs. Tal avaliação é importante para o acompanhamento longitudinal da repercussão do projeto, no que tange aos efeitos da rotina de exercícios. Isso permitirá julgar a efetividade do programa, bem como subsidiar futuras correções e adaptações. Assim, neste estudo, objetivou-se analisar a aptidão físico-funcional e psicológica (nível de depressão e percepção da qualidade de vida) de idosos frequentadores das RALs da cidade do Rio de Janeiro. Testou-se a hipótese de que praticantes regulares de exercícios físicos oferecidos nas RALs exibiriam resultados superiores aos encontrados usualmente em idosos sedentários.

\section{Métodos}

\section{Amostra}

Participaram do estudo 294 voluntários (179 mulheres - 61\%), com idades entre 60 e 86 anos $(68,6 \pm 6,6$ anos; $68,0 \pm 16,5 \mathrm{~kg}, 162,0 \pm 0,1 \mathrm{~cm})$, frequentadores de dez RALs da cidade do Rio de Janeiro (RJ, Brasil) há no mínimo três e, no máximo, 12 meses. Para a seleção da amostra foram adotados os seguintes critérios de exclusão: a) restrições médicas para a prática de exercício físico; b) deficiência motora ou cognitiva impeditiva da aplicação das atividades previstas (questionário e teste de campo); c) problemas osteomioarticulares que comprometessem a realização dos testes; d) frequência inferior a 80\% das sessões de treinamento no período de participação nas atividades.

O estudo foi aprovado pelo Comitê de Ética e Pesquisa da Secretaria Municipal de Saúde e Defesa Civil, da cidade do Rio de Janeiro (Protocolo CAAE: 22065913.5.0000.5279). Todos os voluntários foram devidamente esclarecidos a respeito dos procedimentos e possíveis riscos 
envolvidos no estudo e, então, assinaram um termo de consentimento livre e esclarecido.

\section{Delineamento do estudo}

A coleta de dados foi realizada em três dias, separados por 24 a 48 horas, por uma equipe treinada, no próprio ambiente e horário de prática dos sujeitos. Nas duas primeiras visitas os sujeitos responderam os questionários de Escala de Depressão Geriátrica (EDG) e o Questionário de Qualidade de Vida da Organização Mundial da Saúde para Idosos (WHOQOL-OLD). Na terceira visita, aplicou-se a bateria de testes de aptidão física e funcional proposta por Rikli e Jones ${ }^{5}$.

\section{Avaliação antropométrica}

A massa corporal foi medida em balança digital (Techline, modelo/BAL-10, São Paulo, SP, Brasil); e a estatura, com auxílio de estadiômetro graduado em milímetros Sanny (American Medical do Brasil, São Paulo, SP, Brasil) e aferidas de acordo com as padronizações descritas por Gordon et al. ${ }^{6}$ e Martin et al. ${ }^{7}$, respectivamente. O Índice de Massa Corporal (IMC) foi calculado e considerou-se como baixo-peso IMC $<18,5 \mathrm{~kg} / \mathrm{m}^{2}$, sobrepeso $\mathrm{IMC} \geq 25 \mathrm{~kg} / \mathrm{m}^{2}$ e obesidade IMC $>30 \mathrm{~kg} / \mathrm{m}^{2}$.

\section{Avaliação da depressão e qualidade de vida}

A depressão foi avaliada por meio da Escala de Depressão Geriátrica - EDG. Trata-se de instrumento amplamente utilizado e validado ${ }^{8}$ para diagnóstico de depressão em pacientes idosos, consistindo em 15 perguntas negativas/afirmativas. Um escore igual ou menor a 4 indica ausência de depressão; de 5 a 6 , sensibilidade à depressão; igual ou maior a 7 caracteriza depressão.

A qualidade de vida foi avaliada pelo WHOQUOL - OLD, em versão validada para a língua portuguesa' ${ }^{9}$, considerando-se os seguintes domínios: habilidades sensoriais; autonomia; atividades no passado, presente e futuro; participação social; morte ou morrer; intimidade. $\mathrm{O}$ escores compreendidos entre 4 e 10,9 correspondem a um nível baixo de qualidade vida; entre 11 e 14, a um nível regular, e entre 14,1 e 20, a um nível alto.

\section{Avaliação da aptidõo física funcional}

Para mensuração da aptidão física funcional utilizou-se a bateria de testes proposta por Rikli e Jones ${ }^{5}$, composta pelos seguintes testes: levantar e sentar de uma cadeira e flexão do antebraço (força de membros inferiores e superiores); sentar e alcançar e alcançar atrás das costas (flexibilidade); Timed Up \& Go (agilidade); caminhada de seis minutos (resistência aeróbia).

\section{Programa de exercícios}

As sessões de treinamento foram realizadas três vezes por semana em dias alternados, sendo compostas por equipamentos especificamente desenvolvidos para as RALs, nos quais se efetuaram nove exercícios de força muscular (quatro para membros inferiores, e cinco para superiores), dois exercícios para a resistência aeróbia e dois alongamentos. Os exercícios foram executados em circuito, alternando membros inferiores e superiores, sendo feitas três passagens pelo circuito, com 20 repetições em cada exercício. Os alongamentos foram realizados no início da seção, com duração média de 10 minutos, na faixa de conforto dos participantes. As sessões de treinamento foram de aproximadamente 45 minutos.

\section{Anólise estatística}

Os dados foram analisados por meio de estatística descritiva, e os resultados apresentados sob a forma de média e desvio-padrão, no caso da caracterização dos sujeitos e dos testes de aptidão físico-funcional de Rikli e Jones, e mediana e coeficiente de variação para a Escala de Depressão Geriátrica (EDG) e Questionário de 
Qualidade de Vida da Organização Mundial da Saúde para Idosos (WHOQOL-OLD).

\section{Resultados}

A Tabela 1 apresenta a caracterização dos sujeitos da amostra. Chama-se atenção para o fato de a maioria dos sujeitos ser do sexo feminino e o IMC indicar que a amostra se encontra com sobrepeso.

Os resultados dos testes de aptidão físicofuncional de Rikli e Jones, Escala de Depressão Geriátrica (EDG) e Questionário de Qualidade de Vida da Organização Mundial da Saúde para Idosos (WHOQOL-OLD) estão apresentados na Tabela 2.

\section{Tabela l: Caracterização dos sujeitos}

\begin{tabular}{|c|c|c|c|c|c|}
\hline \multicolumn{2}{|c|}{$\begin{array}{l}\text { Sujeitos } \\
N=294\end{array}$} & \multirow{2}{*}{$\begin{array}{l}\text { Idade } \\
\text { (Anos) }\end{array}$} & \multirow{2}{*}{$\begin{array}{c}\text { Massa } \\
\text { corporal } \\
(\mathrm{kg})\end{array}$} & \multirow{2}{*}{$\begin{array}{l}\text { Estatura } \\
(\mathrm{m})\end{array}$} & \multirow{2}{*}{ IMC } \\
\hline $\mathrm{F}$ & $M$ & & & & \\
\hline $61 \%$ & $39 \%$ & $68,6 \pm 6,6$ & $68,0 \pm 16$ & $1,62 \pm 0,1$ & $26 \pm 6,1$ \\
\hline
\end{tabular}

Fonte: Os autores, 2017.

Levantar e sentar de uma cadeira e flexão do antebraço (força de membros inferiores e superiores); sentar e alcançar e alcançar atrás das costas (flexibilidade); Timed Up \& Go (agilidade); caminhada de seis minutos (resistência aeróbia).

Os resultados obtidos mediante o protocolo de Rikli e Jones ${ }^{5}$ (1999), utilizado para avaliar a aptidão física funcional de idosos, indicam valores nos níveis recomendados em quatro dos seis testes. Somente a flexibilidade dos membros inferiores e superiores e a mobilidade apresentaram-se abaixo dos níveis recomendados.

$\mathrm{O}$ índice de depressão demonstrado pelos sujeitos da pesquisa, também mostrado na Tabela 2, indica que o grupo não apresenta quadro de depressão.

A Tabela 2 traz ainda o resultado obtido nos seis domínios que compõem o WHOQUOL - OLD. Os valores obtidos indicam um nível alto de qualidade de vida dos idosos do grupo investigado em todos os domínios.
Tabela 2: Resultado dos testes

\begin{tabular}{lcccc}
\hline \multicolumn{2}{l}{ Aptidão físico-funcional } & \multicolumn{3}{c}{$\begin{array}{c}\text { Scores de Rikli e } \\
\text { Jones (1999) }\end{array}$} \\
\cline { 4 - 6 } Testes & X & S & F & M \\
\hline Sentar e levantar & $14,9 \uparrow$ & 2,3 & $11-16$ & $12-18$ \\
$\begin{array}{l}\text { Flexão de cotovelo } \\
\text { 6 Minutos de }\end{array}$ & $17,0 \uparrow$ & 2,6 & $12-18$ & $15-21$ \\
$\begin{array}{l}\text { caminhada } \\
\text { Sentar e alcançar }\end{array}$ & $587,6 \uparrow$ & 57,8 & $500-635$ & $560-700$ \\
Alcançar as costas & $-7,6 \downarrow$ & 7,1 & $-0,5-4,5$ & $-3,0-3$ \\
$\begin{array}{l}\text { Time Up and Go } \\
\text { (agilidade) }\end{array}$ & $8,6 \downarrow$ & 24,4 & $6,4-4,8$ & $5,7-4,3$ \\
\hline
\end{tabular}

$\uparrow$ Acima do valor mínimo recomendado para ambos os sexos

$\downarrow$ Abaixo do valor mínimo recomendado para ambos os sexos

F: Feminino; M: Masculino; X: Média; S: Desvio-padrão

Escala de Depressão Geriátrica - EDG

\begin{tabular}{lcc}
\hline Teste & MD & CV\% \\
\hline \multicolumn{1}{c}{ Índice obtido $1,0^{*}$} & 91,7 \\
\hline${ }^{*}$ Ausência de depressão $(\leq 4)$ & \\
MD: Mediana & \\
CV\%: Coeficiente de Variação &
\end{tabular}

WHOQOL-OLD

\begin{tabular}{lcc}
\hline Teste & MD & CV\% \\
\hline Habilidades sensoriais & $16,0 \uparrow$ & 27,4 \\
Autonomia & $15,0 \uparrow$ & 22,2 \\
Atividades passadas, & $16,0 \uparrow$ & 18,9 \\
presentes e futuras & $16,0 \uparrow$ & 20,3 \\
Participação social & $15,0 \uparrow$ & 28,4 \\
Morte ou morrer & $16,0 \uparrow$ & 21,4 \\
Intimidade &
\end{tabular}

$\uparrow$ Nível alto (14,1 a 20)

MD: Mediana

CV\%: Coeficiente de Variação

Fonte: Os autores, 2017.

\section{Discussão}

Com o propósito de analisar a aptidão físico-funcional e psicológica de idosos frequentadores das RALs da cidade do Rio de Janeiro, foram levantados dados que caracterizassem a amostra, que cabem aqui serem analisados.

É interessante ressaltar a predominância de indivíduos do sexo feminino (Tabela 1) na atual pesquisa. Essa característica é típica na maioria dos estudos desenvolvidos com idosos. Vagetti et al. ${ }^{10}$, ao realizarem uma revisão sistemática objetivando verificar informações sobre 
a associação de atividade física com a qualidade de vida em idosos, encontraram 42 trabalhos, dos quais $26,19 \%$ foram feitos exclusivamente com mulheres; e a grande parte dos demais, com um maior número de participantes do sexo feminino. Segundo esses autores ${ }^{10}$, isso se deve, possivelmente, porque as mulheres possuem maior expectativa de vida, maior nível de apoio social, são mais propensas a buscar novas fontes de apoio, possuem maior capacidade de formar relações afetivas, e menor vulnerabilidade biológica, além da taxa de doenças letais ser maior entre homens.

Para identificar o perfil relacionado com o estado nutricional da amostra, dado importante por estar relacionado com a predisposição para o desenvolvimento de muitas doenças crônico degenerativas, como o diabetes e a hipertensão ${ }^{11}$, o IMC foi calculado. Seus valores estão apresentados na Tabela 1 e indicam que os sujeitos se encontravam com sobrepeso no momento da coleta, sugerindo que a atividade física praticada pode não estar contribuindo para o controle do peso corporal dos idosos. No entanto, muitos estudos desenvolvidos com idosos praticantes de diferentes atividades físicas obtiveram resultados similares aos da atual amostra, como os de Cunha et al. ${ }^{12}$ e Matos et al. ${ }^{13}$.

Cunha et al. ${ }^{12}$, em estudo realizado com idosas participantes de atividades físicas desenvolvidas em um Posto de Saúde da Família (PSF) da cidade de Juazeiro do Norte, demonstraram que os exercícios físicos realizados foram suficientes para provocar a diminuição nos valores médios de massa corporal das idosas, consequentemente, no IMC, mas não o suficiente para levá-las a atingir níveis considerados normais para o estado nutricional. Já Matos et al. ${ }^{13}$ avaliaram os efeitos de oito semanas de treinamento funcional sobre a autonomia funcional de idosas, e sua amostra também apresentou valores de IMC referentes a sobrepeso. Esses resultados se aproximam aos encontrados neste estudo, sugerindo que o sobrepeso em idosas pode ser uma característica incidente, tendo em vista o aumento da gordura corporal e a diminuição da massa magra advindos do processo de envelhecimento ${ }^{14}$.

O nível de aptidão física vem sendo reconhecido com um indicador que tem fortes relações com a manutenção da saúde e qualidade de vida do idoso ${ }^{2}$. Diversos estudos vêm reforçando a ideia de que manter um estilo de vida ativo, consequentemente bom nível de aptidão física, contribui para a manutenção da autonomia funcional ${ }^{13,15}$ e para a prevenção de doenças crônicodegenerativas ${ }^{2}$, bem como para aquelas de fundo psicológico, como a depressão ${ }^{16}$, colaborando para um envelhecer com melhor qualidade de vida $^{2}$. Sendo assim, diante do objetivo nesta pesquisa, avaliar o perfil da aptidão física funcional dos idosos que frequentam as RALs da cidade do Rio de Janeiro-RJ/Brasil é relevante para se verificar a eficácia da proposta de treinamento físico ofertado a essa população pela prefeitura.

Mesmo sem um maior controle da intensidade da carga de treinamento, os resultados demonstraram que o programa parece ser eficaz para a manutenção ou desenvolvimento da força e resistência dos membros inferiores e superiores de idosos. Esse resultado pode ser explicado pelos achados de Silva et al. ${ }^{4}$, os quais, pela metanálise, concluíram que o tempo de treinamento parece ser a variável mais importante para o desenvolvimento da força muscular, do que as demais variáveis as quais combinadas compõem seu treinamento (Número de repetições, número séries, intensidade da carga). Desse modo, o simples fato de os idosos estarem estimulando a força muscular de forma sistematizada e com adesão ao treinamento proposto pelo programa investigado está sendo positivo para a variável em questão.

Esse achado é significativo, uma vez que a força e a resistência muscular se apresentam como componentes da aptidão física de extrema importância para a autonomia funcional de ido$\operatorname{sos}^{17}$, uma vez que sua diminuição ao longo do processo de envelhecimento influencia negativamente na capacidade de execução das atividades de vida diária (AVD) ${ }^{4}$, além disso, guardam forte relação com a manutenção da postura ${ }^{18} \mathrm{e}$ 
de um padrão de marcha adequados ${ }^{19}$, fatores que influenciam sobremaneira a prevenção de quedas ${ }^{18,19}$.

Outro dado igualmente importante ressaltado no atual estudo foi o fato de a resistência aeróbia também apresentar resultados acima dos níveis mínimos recomendados. Esse componente da aptidão física vem sendo apontado como de extrema relevância para a saúde dos seres humanos, dada a relação existente entre o nível de aptidão cardiorrespiratória e a proteção desse sistema contra doenças crônico-degenerativas, como a hipertensão e a insuficiência cardíaca $^{15}$, muito comuns em idades avançadas ${ }^{20}$, que acabam por vezes também contribuindo para a redução da autonomia funcional do idoso.

Diferentemente do comportamento da força muscular e da resistência cardiorrespiratória, destaca-se que a flexibilidade dos membros inferiores e superiores e a agilidade ficaram aquém do mínimo desejado. Esse dado é importante, pois ambos os componentes são necessários para a manutenção da capacidade funcional de idosos ${ }^{3}$. No caso da flexibilidade, ela é necessária para a realização da maioria das tarefas cotidianas presentes na rotina de idosos ${ }^{21}$.

Os resultados relativos à flexibilidade e à agilidade se afastam da maioria dos estudos, os quais indicam que diferentes tipos de exercícios físicos colaboram para o aperfeiçoamento e/ou manutenção dessas qualidades físicas ${ }^{22}$, no entanto, se aproxima parcialmente de algumas pesquisas encontradas na literatura, como as de Todde et al. ${ }^{23}$ e Tsuji et al. ${ }^{24}$.

Todde et al. ${ }^{23}$ realizaram um estudo controlado randomizado com idosos sedentários e avaliaram a autonomia funcional por meio do Senior Fitness Test. A amostra foi submetida a 12 semanas de intervenção de um protocolo de exercícios mistos, realizado a uma intensidade controlada, entre $60 \%$ e $84 \%$ da frequência cardíaca de reserva. Verificaram-se melhoras significativas em todos os parâmetros do instrumento, exceto no teste de agilidade. Tal resultado pode ser comparado ao desta investigação, em relação ao parâmetro agilidade, apesar da amostra não ser sedentária e não haver nenhum tipo de controle da intensidade das atividades realizadas no Rio ao Ar Livre.

Já Tsuji et al. ${ }^{24}$ encontraram melhora significativa da agilidade e da flexibilidade de membros inferiores de idosos avaliados, respectivamente, pelo teste Sentar e Alcançar e do Chair Sit-\&-Reach (agilidade), após 30 minutos de exercícios estáticos em uma plataforma vibratória. Todavia, esses resultados foram significativos apenas agudamente, não sendo encontradas melhoras em longo prazo.

Os resultados insatisfatórios apresentados pela amostra do atual estudo para a flexibilidade e a agilidade podem indicar que o modelo de treinamento do Rio Ar Livre não seja eficaz para o desenvolvimento dessas capacidades físicas.

Como não foram encontrados na literatura estudos que investigassem programas de exercícios físicos similares ao desta pesquisa, análises comparativas dos achados ficaram dificultadas. No entanto, pode-se afirmar que os resultados obtidos corroboram o que já está muito bem descrito na literatura, que os exercícios físicos regulares contribuem para a manutenção da aptidão física funcional de indivíduos mais velhos ${ }^{2}$.

Perder a independência para realização das AVDs, como consequência da diminuição da aptidão física funcional, pode ter ainda para o idoso, graves implicações que acabam por provocar desajustes psicossociais ${ }^{10}$, ocasionando doenças psicológicas, como a depressão ${ }^{25}$.

A depressão é prevalente entre adultos mais velhos e está associada à significativa morbidade e aumento do risco de mortalidade ${ }^{26}$. Exercícios físicos regulares têm sido apontados como tratamento eficaz da depressão ${ }^{26,27}$. Nesse sentido, para verificar a eficácia da proposta de treinamento físico ofertada pelas RALs investigadas, achou-se importante avaliar essa variável.

O índice de depressão demonstrado pelos sujeitos da pesquisa indicou que o grupo não apresenta quadro de depressão, o que pode corroborar as evidências apontadas pela literatura $^{26,27}$. Em trabalhos de revisão sistemática, 
Barbour e Blumenthal ${ }^{26}$ sugerem que a prática regular de exercícios físicos leva a uma redução dos sintomas depressivos. Mather et al. ${ }^{27}$, em um estudo experimental, reforçam essas evidências ao encontrarem diminuição de 30\% dos sintomas depressivos apresentados por idosos submetidos a dez semanas de prática de exercícios físicos.

A Qualidade de Vida vem sendo definida como a percepção do indivíduo de sua posição na vida, no contexto da cultura e sistema de valores nos quais vive e em relação a seus objetivos, suas expectativas, seus padrões e suas preocupações ${ }^{28}$. Sendo assim, estudos demonstram que a dependência para realização das AVDs e os níveis elevados de depressão ${ }^{25}$ interferem negativamente nessa percepção. Por isso, a prática regular de atividade física vem sendo reconhecida como uma estratégia importante para a manutenção da qualidade de vida do idoso ${ }^{2}$.

Neste estudo, em todos os domínios do WHOQUOL - OLD, os sujeitos investigados demonstraram nível alto de percepção da qualidade de vida. Esse resultado reforça a hipótese de que a prática regular de exercícios físicos contribui para uma percepção aumentada da qualidade de vida de idosos, conforme proposto por Costa et al..$^{29}$, ao compararem o nível de qualidade de vida de idosos praticantes e não praticantes de atividade física, quando também encontraram uma pontuação significativamente menor em todos os domínios do WHOQUOL OLD no grupo de não praticantes. Assim como na atual investigação e na de Costa et al. ${ }^{29}$, outros autores já chegaram à mesma conclusão ${ }^{30}$.

\section{Conclusão}

Em que pese o fato de o atual estudo se configurar como ex post facto, pode-se dizer que as RALs parecem se constituir como uma estratégia positiva, ao menos para o grupo investigado, uma vez que a maioria das variáveis avaliadas se apresenta dentro de padrões esperados para a população de idosos.

\section{Referências}

1. World Health Organization (WHO). Global Health and Aging. NIH Publication N ${ }^{\circ}$ 11-7737, Oct, 2011.

2. American College of Sports Medicine Position Stand (ACSM). Exercise and physical activity for older adults. Med Sci Sports Exerc. 2009;41(7):1510-3.

3. Secretaria Especial de Envelhecimento e Qualidade de Vida (SESQV). Rio Ar Livre (RAL) [Texto explicativo] In: Prefeitura do Rio de Janeiro. Rio de Janeiro, 2015. Disponível em: http://www.rio.rj.gov. br/web/sesqv/exibeconteudo?id=5584533. Acesso em: 30 out. 2015 .

4. Silva NL, Oliveira RB, Fleck SJ, Leon ACMP, Farinatti $\mathrm{P}$. Influence of strength training variables on strength gains in adults over 55 years-old: a meta-analysis of dose-response relationships. J Sci Med Sport. 2014;17(3):337-44.

5. Rikli R, Jones J. Development and validation of a functional fitness test for community-residing older adults. J Aging Phys Act. 1999;7:129-61.

6. Gordon C, Chunlea WC, Roche AF. Stature, recumbent length, and weight. Champaign: Human Kinetics; 1988.

7. Martin AD, Carter JEL, Hendy KC, Malina RM Segment lengths. Champaign: Human Kinetics; 1988.

8. De Waala MWM, Van Der Weelea GM, Van Der Mastb RC, Assendelfta WJJ, Gusseklooa J. The influence of the administration method on scores of the 15-item Geriatric Depression Scale in old age. Psychiatry Res. 2012 May 30;197(3):280-4.

9. Fleck MP, Chachamovich E, Trentini C. Development and validation of the Portuguese version of the WHOQOL-OLD module. Rev Saúde Públ. 2006;40(5):785-91.

10. Vagetti GC, Barbosa Filho VC, Moreira NB, Oliveira V, Mazzardo O, Campos W. Association between physical activity and quality of life in the elderly: a systematic review, 2000-2012. Rev Bras Psiquiatr. 2014;36(1):76-88.

11. Wong KA, Kataoka-Yahiro MR. Nutrition and diet as it relates to health and well-being of Native Hawaiian Kūpuna (elders): a systematic literature review. J Transcult Nurs. 2017;28(4):408-22.

12. Cunha RCL, Fortes MSR, Ferreira MA, Bezerra JCP, Silva JMFL, Graup $S$ et al. Efeitos de um programa de caminhada sob os níveis de autonomia funcional de idosas monitoradas pelo Programa de Saúde da Família. Rev Bras Geriatr Gerontol. 2010;13(2):255-65. 
13. Matos DG, Mazini Filho ML, Moreira OC, Oliveira CE, Venturini GR, Silva-Grigoletto ME et al. Effects of eight weeks of functional training in the functional autonomy of elderly women: a pilot study. J Sports Med Phys Fitness. 2017;57(3):272-7.

14. CHOI KM. Sarcopenic and sarcopenic obesity. Korean J Intern Med. 2016;31:1054-60.

15. Oliveira Brito LV, Maranhão Neto GA, Moraes H, Emerick RF, Deslandes AC. Relationship between level of independence in activities of daily living and estimated cardiovascular capacity in elderly women. Arch Gerontol Geriatr. 2014;59(2):367-71.

16. Blanchet S, Chikhi S, Maltais D. The benefits of physical activities on cognitive and mental health in healthy and pathological aging. Geriatr Psychol Neuropsychiatr Vieil. 2018;16(2):197-205.

17. Hwang AC, Zhan YR, Lee WJ, Peng LN, Chen LY, Lin MH et al. Higher daily physical activities continue to preserve muscle strength after midlife, but not muscle mass after age of 75 . Medicine (Baltimore). 2016;95(22):e3809.

18. Fan Y, Li Z, Han S, Lv C, Zhang B. The influence of gait speed on the stability of walking among the elderly. Gait Posture. 2016;47:31-6.

19. Rosenlund S, Holsgaard-Larsen A, Overgaard S, Jensen $C$. The gait deviation index is associated with hip muscle strength and patient-reported outcome in patients with severe hip osteoarthritis-a crosssectional study. PLoS One. 2016;11(4):e0153177.

20. Benjamin EJ, Muntner P, Alonso A, Bittencourt MS, Callaway CW, Carson AP, et al. Heart Disease and Stroke Statistics-2019 Update: a report from the American Heart Association. Circulation. 2019;139:00-00.

21. Penatti VV, Gobbo LA. O impacto dos treinamentos com pesos, aeróbio, de flexibilidade e de equilíbrio na aptidão funcional e qualidade de vida dos idosos. Mais 60: Estudos sobre Envelhecimento. 2015;26(63):32-49.
22. Bullo V, Bergamin M, Gobbo S, Sieverdes JC, Zaccaria M, Neunhaeuserer D, Ermolao A. The effects of Pilates exercise training on physical fitness and wellbeing in the elderly: a systematic review for future exercise prescription. Prev Med. 2015;75:1-11.

23. Todde F, Melis F, Mura R, Pau M, Fois F, Magnani $\mathrm{S}$ et al. A 12-week vigorous exercise protocol in a healthy group of persons over 65: study of physical function by means of the Senior Fitness Test. Biomed Res Int. 2016;2016:1-6.

24. Tsuji T, Kitano N, Tsunoda K, Himori E, Okura T, Tanaka K. Short-term effects of whole-body vibration on functional mobility and flexibility in healthy, older adults: a randomized crossover study. J Geriatr Phys Ther. 2014;37(2):58-64.

25. Teixeira AR, Wender MH, Gonçalves AK, Freitas C. LR, Santos AMPV, Soldera CLC. Dizziness, physical exercise, falls, and depression in adults and the elderly. Int Arch Otorhinolaryngol. 2016;20:124-131.

26. Barbour KA, Blumenthal JA. Exercise training and depression in older adults. Neurobiol Aging. 2005;26(Suppl 1):119-23.

27. Mather AS, Rodriguez C, Guthrie MF, McHarg AM, Reid IC, McMurdo ME. Effects of exercise on depressive symptoms in older adults with poorly responsive depressive disorder: randomized controlled trial. Br J Psychiatry. 2002;180:411-5.

28. Fuchs J, Scheidt-Nave C, Hinrichs T, Mergenthaler A, Stein J, Riedel-Heller SG, Grill E. Indicators for healthy ageing -A debate. Int J Environ Res Public Health. 2013;10:6630-44.

29. Costa FR, Rodrigues FM, Prudente COM, Souza IF. Qualidade de vida de idosos participantes e não participantes de programas públicos de exercícios físicos. Rev Bras Geriatr Gerontol. 2018;21(1):24-34.

30. Ferretti F, Beskow GCT, Slaviero RC, Ribeiro CG. Análise da qualidade de vida em idosos praticantes e não praticantes de exercício físico regular. Estud Interdiscipl Envelhec. 2015; 20(3):729-43. 